\title{
Ancestral mesodermal reorganization and evolution of the vertebrate head
}

\author{
Takayuki Onai ${ }^{{ }^{*}}$, Toshihiro Aramaki ${ }^{2}$, Hidehiko Inomata ${ }^{3}$, Tamami Hirai ${ }^{1}$ and Shigeru Kuratani ${ }^{1}$
}

\begin{abstract}
Introduction: The vertebrate head is characterized by unsegmented head mesoderm the evolutionary origin of which remains enigmatic. The head mesoderm is derived from the rostral part of the dorsal mesoderm, which is regionalized anteroposteriorly during gastrulation. The basal chordate amphioxus resembles vertebrates due to the presence of somites, but it lacks unsegmented head mesoderm. Gastrulation in amphioxus occurs by simple invagination with little mesodermal involution, whereas in vertebrates gastrulation is organized by massive cell movements, such as involution, convergence and extension, and cell migration.

Results: To identify key developmental events in the evolution of the vertebrate head mesoderm, we compared anterior/posterior (A/P) patterning mechanisms of the dorsal mesoderm in amphioxus and vertebrates. The dorsal mesodermal genes gsc, bra, and delta are expressed in similar patterns in early embryos of both animals, but later in development, these expression domains become anteroposteriorly segregated only in vertebrates. Suppression of mesodermal involution in vertebrate embryos by inhibition of convergence and extension recapitulates amphioxus-like dorsal mesoderm formation.
\end{abstract}

Conclusions: Reorganization of ancient mesoderm was likely involved in the evolution of the vertebrate head.

Keywords: Amphioxus, Head mesoderm, Vertebrate body plan, Somites

\section{Introduction}

How the highly complex vertebrate head-composed of brain, head muscles, and skull-evolved from nonvertebrate ancestors is a fundamental question in current evolutionary and developmental biology [1-3]. Recent comparative studies of the cephalochordate amphioxus and vertebrates suggest that a region homologous to the vertebrate fore/mid/hind brain is also present in the rostral part of the central nervous system (CNS) of amphioxus [4-6]. Amphioxus is a basal chordate that has somites extending to the rostral end of the body, and is considered the best proxy for understanding the origin of the vertebrate body plan [7].

The homology between amphioxus and the vertebrate CNS indicates that the unsegmented vertebrate head mesoderm evolved directly from the rostral somites of amphioxus [8]. Expression of $e n$ in the rostral somites of

\footnotetext{
*Correspondence: tonai@cdb.riken.jp

'Kuratani Evolutionary Morphology Laboratory, RIKEN Center for Developmental Biology, 2-2-3 Minatojima-minamimachi, Chuo-ku, Kobe 650-0047, Japan

Full list of author information is available at the end of the article
}

amphioxus (Branchiostoma floridae) and en2 in the ventral part of the mandibular head mesoderm of shark (Scyliorhinus torazame) embryos supports this hypothesis $[9,10]$. However, Bfpax3/7, a homologue of pax3 that serves as a somite marker in vertebrates, is expressed in the rostral somites, suggesting that the vertebrate head mesoderm did not evolve by simple modification of rostral somites of an amphioxus-like ancestor, but rather by fundamental reorganization that occurred in the dorsal mesoderm $[2,10]$.

During embryogenesis, the vertebrate head mesoderm derived from the rostral part of the dorsal mesoderm is regionalized along the $\mathrm{A} / \mathrm{P}$ axis by a gradient of $\mathrm{Wnt} / \beta$-catenin signalling $[11,12]$. In the regionalization of the dorsal mesoderm, downstream regional marker genes of $\mathrm{Wnt} / \beta$-catenin signalling are expressed in the progenitor domains; gsc is expressed in the head mesoderm, whereas bra is expressed in the presumptive notochord during the late-gastrula stage [13, 14]. Additionally, in the trunk mesoderm, delta expression is detected in the presumptive somite region $[15,16]$. Previous functional studies have 
shown that overexpression of Xenopus laevis dkk1 (negative regulator of Wnt/ $\mathrm{W}$-catenin signalling) expands the gsc expression domain posteriorly in Xenopus embryos, whereas bra expression is activated by Wnt/ $\beta$-catenin signalling $[11,17,18]$. Additionally, delta has an essential role in somitogenesis, and is under the control of Wnt/ $\beta$-catenin signalling [19].

In amphioxus, gsc and bra are co-expressed in the presumptive notochordal region at the gastrula stage $[20,21]$. The presumptive somite marker delta is expressed in the first and second somites in the lategastrula stage [22]. Loss of gsc expression in the notochord and gain of gsc expression in the head mesoderm of vertebrates compared with amphioxus indicates that $\mathrm{A} / \mathrm{P}$ re-arrangement of mesodermal gene expression occurred in the lineage of vertebrates. Excessive $W n t / \beta$-catenin signalling in amphioxus embryos induced by inhibition of GSK-3 $\alpha / \beta$ does not affect the expression of regional marker genes of the dorsal mesoderm, such as bra and $f g f 8 / 17 / 18$, during the gastrula stage [23]. This suggests that, unlike in vertebrates, $\mathrm{Wnt} / \beta$-catenin signalling does not play a role in dorsal mesoderm regionalization in amphioxus. If vertebrate embryos did evolve a rearrangement of gene expression in the dorsal mesoderm to generate the head mesodermal region, what was the key developmental event in this process? We consider that rearrangement of gene expression in the vertebrate dorsal mesoderm from an ancestral chordate evolved through a novel mesodermal cell movement present in vertebrates.

Amphioxus gastrulation occurs through simple invagination, with little mesodermal involution of the outer layer [24], whereas in vertebrates, an overt involution takes place, as observed in Xenopus and lamprey [25-27]. Thus, uniquely in amphioxus and distinct from the case in vertebrates, there is nearly no change in the relative positions of the ectoderm and mesoderm. However, it remains unclear how mesodermal involution affects $\mathrm{A} / \mathrm{P}$ patterning of the dorsal mesoderm and how this change has led to two distinct types of rostral mesoderm in amphioxus and vertebrates.

To explore the molecular background of vertebrate head mesoderm evolution, we first investigated the developmental stage at which the overall molecular topography of the dorsal mesoderm becomes distinctly different between amphioxus and vertebrates. We also examined whether mesodermal involution is important for dorsal mesoderm regionalization in vertebrate embryos, as a possible developmental factor that gave rise to vertebrate head mesoderm. Finally, we examined whether the genetic program for mesodermal involution is present in amphioxus. We hypothesize that vertebrate head mesoderm evolved from an amphioxus-like ancestral mesoderm through anteroposterior reorganization of the genetic developmental architecture.

\section{Materials and methods}

Sources of amphioxus, lamprey, and shark embryos

In the summer breeding season, adult amphioxus (B. floridae) were collected from Old Tampa Bay, FL, USA. The in vitro fertilization and culturing of the embryos were conducted as described [28]. Adult amphioxus (Branchiostoma japonicum) were collected from the ocean near Amakusa Island, Kumamoto, Japan. Adult lampreys (Lethenteron japonicum) were collected from the Miomote River in Niigata, Japan and the Shirubetu River in Hokkaido, Japan during the spring breeding season. In vitro fertilization was performed as described previously [29]. Adult sharks (S. torazame) were collected from Nakaminato Bay, Ibaraki, Japan in October and maintained in a seawater tank at $16{ }^{\circ} \mathrm{C}$. Eggs were obtained from the adult females and maintained in the seawater tank until they developed to the described stages [30].

\section{In situ hybridization}

Whole-mount in situ hybridization of amphioxus, lamprey, shark, and Xenopus embryos was performed as described in previous studies [10, 29, 31]. For section in situ hybridization of Xenopus embryos, larval-stage embryos were fixed using MEMFA for $2 \mathrm{~h}$ at room temperature then section in situ hybridization was performed as described [32]. For fluorescence in situ hybridization, the protocol used for amphioxus wholemount in situ hybridization [23] was applied and an antibody (Anti-DIG-POD, Roche, Basel, Switzerland) and TSA system (PerkinElmer, Waltham, MA, USA) were used. Cellmask deep red (Life Technologies, Carlsbad, CA, USA) (1/1000) and DAPI (Invitrogen, Carlsbad, CA, USA) (1/1000) were used to stain the plasma membrane or nuclei. A Zeiss LSM 780 (Zeiss, Jena, Germany) was used to collect confocal images.

\section{Plasmid construction and gene markers}

The sequences of Bfdkk1/2/4 [21], Bfgsc [21], Bfbra [20], Bfwnt8 [21], Bfdelta [22], Stdkk1 (KF551566), Stgsc (KF564642), Stdelta (KF551567), Stbra (KF551568), and Stwnt8 (KF551569) were amplified by PCR and cloned into a TOPO cloning vector. Xldkk1-pCS2 (NM_001085592), Xlgsc-pCS2 (NM_001087809), Xldelta2-pCS2 (NM_001086082), Xlbra-pSP64 (M77243.1), Xlwnt8-pCS2 (NM_001088168), and XlmyoD-pCS2 (NM_001085897) were gifts from Dr. Yoshiki Sasai from RIKEN CDB, Japan. Xltbx1 (NM_001090445) was amplified by PCR and cloned into the pCR-II TOPO vector. Ljgsc (KF551572), Ljdelta (KF564639), Ljbra (AB501127), and Ljwnt8 (KF551570) were amplified 
by PCR and cloned into a TOPO cloning vector. $\mathrm{Xl} d d 1$-myc-pCS2, myc-XldshdelDEP-pCS2, and Bfrnd1pCS2 were linearized using NotI and transcribed using SP6 polymerase (mMessage mMachine, Ambion, Austin, TX, USA).

\section{Xenopus experiments}

Embryos were staged according to the Normal Table published by Nieuwkoop and Faber [33]. The mRNA, in $1 \times$ modified Barth's saline, was injected into the embryos using a fine glass capillary tube and a pressure injector (Narishige, Tokyo, Japan). The embryos were then transferred into 0.1× Barth's saline until further manipulation or harvest. For histological analysis, the embryos were fixed using Bouin's fixative and then dehydrated and embedded in paraffin. Sections (6- $\mu$ m-thick) were cut and stained with haematoxylin and eosin. The sequence of the Xlrnd1-morpholino antisense oligonucleotide (MO) has been published [34]. For the dorsal marginal zone assay, embryos were dissected at the early-gastrula stage (stage 10) and cultured in $1 \times$ low-calcium magnesium Riner's supplemented with $0.2 \%$ bovine serum albumin until stage 19.

\section{Immunostaining of amphioxus and Xenopus embryos}

Early-neurula-stage amphioxus embryos were fixed using $4 \%$ paraformaldehyde in MOPS buffer at $4{ }^{\circ} \mathrm{C}$ overnight, and immunocytochemistry was performed as described [35], using a primary antibody against $\beta$-catenin (diluted 1:800 for Xenopus embryos and 1:400 for amphioxus embryos; C-2206, Sigma, St. Louis, MO, USA), a secondary Alexa Fluor 488-conjugated antibody (1:400, Invitrogen), and DAPI (1:1000, Invitrogen). Whole embryos and histological sections were imaged using a Zeiss LSM 710 confocal microscope and a Zeiss Axio Zoom V16 microscope, respectively.

\section{Phylogenetic tree analysis}

Phylogenetic trees were constructed with MEGA5 software [36] using the maximum-likelihood method with 1000 bootstrap reiterations. All sequences were aligned using Clustal W (http://www.clustal.org/clustal2/).

\section{Alignment analysis of FIrt3}

For Flrt3 protein alignment analysis, we used the multiple alignment tool in Genetyx-Mac version 16.0.7 (http:// www.genetyx.co.jp/products/genetyx_mac_16/index.html).

\section{Accession numbers}

The sequences of the novel genes isolated were deposited in GenBank with following accession numbers. Ljgsc (KF551572), Ljdelta1 (KF564639), Ljwnt8 (KF551570), Stdkk1 (KF551566), Stgsc (KF564642), Stbrachyury (KF551568), Stdelta1 (KF551567), and Stwnt8 (KF551569).

\section{Results and discussion}

Genetic topography of the vertebrate dorsal mesoderm evolved through A/P expression domain shift of amphioxus mesodermal genes

In the dorsal mesoderm of amphioxus embryos, somites and notochord are regionalized during gastrulation, and an equivalent structure of the vertebrate unsegmented head mesoderm is thought to be absent in amphioxus [7] (Fig. 1). The dorsal mesoderm of vertebrates such as Xenopus is formed by massive mesodermal involution during the early-gastrula stage, which is not present in amphioxus embryos and is regionalized into head mesoderm, notochord, and somites [27, 37] (Fig. 1), suggesting that genetic programs govern differences between amphioxus and vertebrate mesodermal formation. We thus examined the molecular topography of the dorsal mesoderm in amphioxus and Xenopus. We first compared the mid-gastrula stage of amphioxus embryos and stage 10.5 Xenopus embryos because the orientation of dorsal mesodermal tissue is approximately parallel to the $\mathrm{A} / \mathrm{P}$ body axis in both species, and thus comparable at these stages [33] (Additional file 1: Figure S1). The key regional genes include gsc (head mesoderm), bra (notochord), delta (somite), wnt8 (somite) and dkk1 (head mesoderm, somite).

By the mid-gastrula stage, all genes examined were expressed around the blastopore and showed similar patterns in amphioxus and vertebrates (Fig. 2a and b). However, by the late-gastrula stage of Xenopus, the expression domains of the regional marker genes became separated anteroposteriorly, with the gsc expression domain barely overlapping with those of delta, wnt8, and bra as the head and trunk mesodermal identities became distinct (Fig. 2c-f). These dynamic shifts were also observed in basal vertebrates, such as the lamprey (L. japonicum) and shark ( $S$. torazame) (Additional file 1: Figures S2, S3 and Table S1). During the late-gastrula stage, the presomitic mesodermal region became distinct around the blastopore, and $d k k 1 / 2 / 4$, delta, bra, and wnt8 were co-expressed in Xenopus presomitic mesoderm (Fig. 2d). In amphioxus, somites were found to form directly from the tail bud, and the expression of $d k k 1 / 2 / 4$, delta, bra, and wnt8 largely overlapped in the prospective tail bud region (Fig. 2c). These results suggest that during gastrula stages, the mesodermal genes segregate anteroposteriorly only in vertebrates, whereas in amphioxus, these genes overlap considerably (Fig. 2f).

\section{Interference of mesodermal involution promotes an} amphioxus developmental mode in Xenopus embryos

Just after the stage in which mesodermal gene expression arrangements were similar in amphioxus and Xenopus, the dorsal mesoderm in Xenopus spread anteriorly to the blastocoel to form the head mesoderm (Fig. 3a and b). 


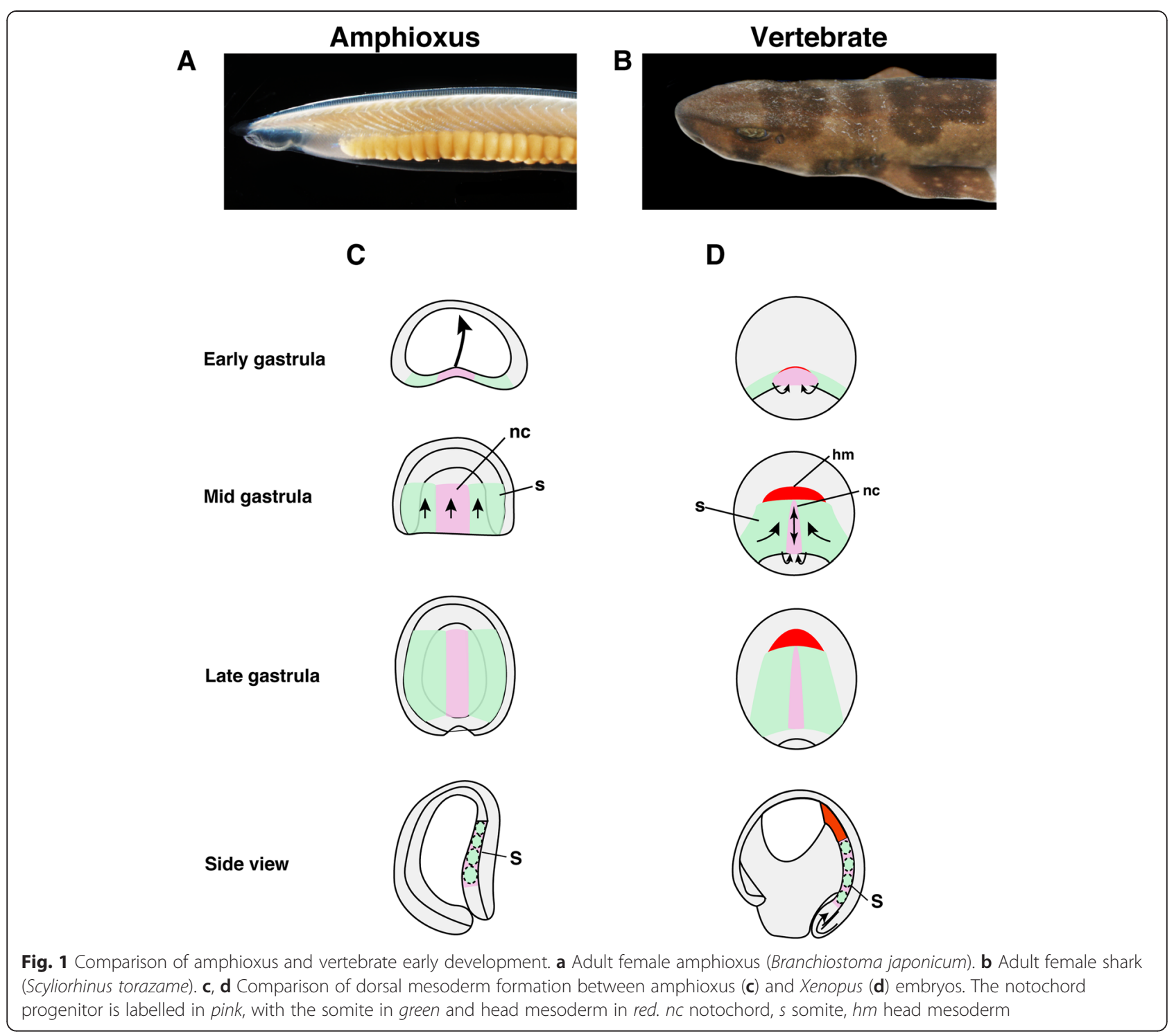

However, in amphioxus, the relative increase in mesodermal size was much lower than that in vertebrates (Fig. 3c). This suggests that, in vertebrates, the dynamic mesodermal gene shift is achieved by increasing the mesoderm, which is primarily dictated by mesodermal cell movements (e.g. involution, convergence and extension) (Fig. 3c). During gastrulation, mesodermal involution is controlled by convergence and extension of the dorsal axial mesoderm in vertebrates [38, 39]. To determine whether mesodermal involution is essential for the mesodermal gene shift, we suppressed convergent extension by inhibiting the Wnt planar cell polarity (PCP) signalling pathway, (a key signal transduction pathway in convergence and extension) [40, 41]. For the loss of function study of Wnt/PCP signalling, we injected Xldd1 (a dominant-negative form of Xldsh; [42, 43]) mRNA into Xenopus embryos [26].
In the dye only-injected control embryos, labelled cells migrated anteriorly and expressed gsc but not delta-2 or bra (Fig. 3d, e, g and i). In Xldd 1 mRNA-injected embryos, however, labelled cells did not migrate anteriorly, but remained close to the blastopore, and gsc, delta-2, and bra were not separated anteroposteriorly as observed in the control embryos during the late-gastrula stage (Fig. 3f, $h$ and $j$ ). Additionally, the size of the dorsal mesoderm was much smaller in the Xldd1 mRNAinjected embryos compared with control embryos (Fig. $3 \mathrm{e}-\mathrm{j}$ ), indicating that the developmental sequences of the dorsal mesoderm were somewhat similar to those in amphioxus. Microinjection of XldshdelDEP mRNA, a mutant $d s h$ that specifically inhibits the Wnt/PCP signalling pathway [44], indicated that the effect of Xldd1 injection resulted from suppression of the Wnt/PCP signalling pathway (Additional file 1: Figure S4A-F). 

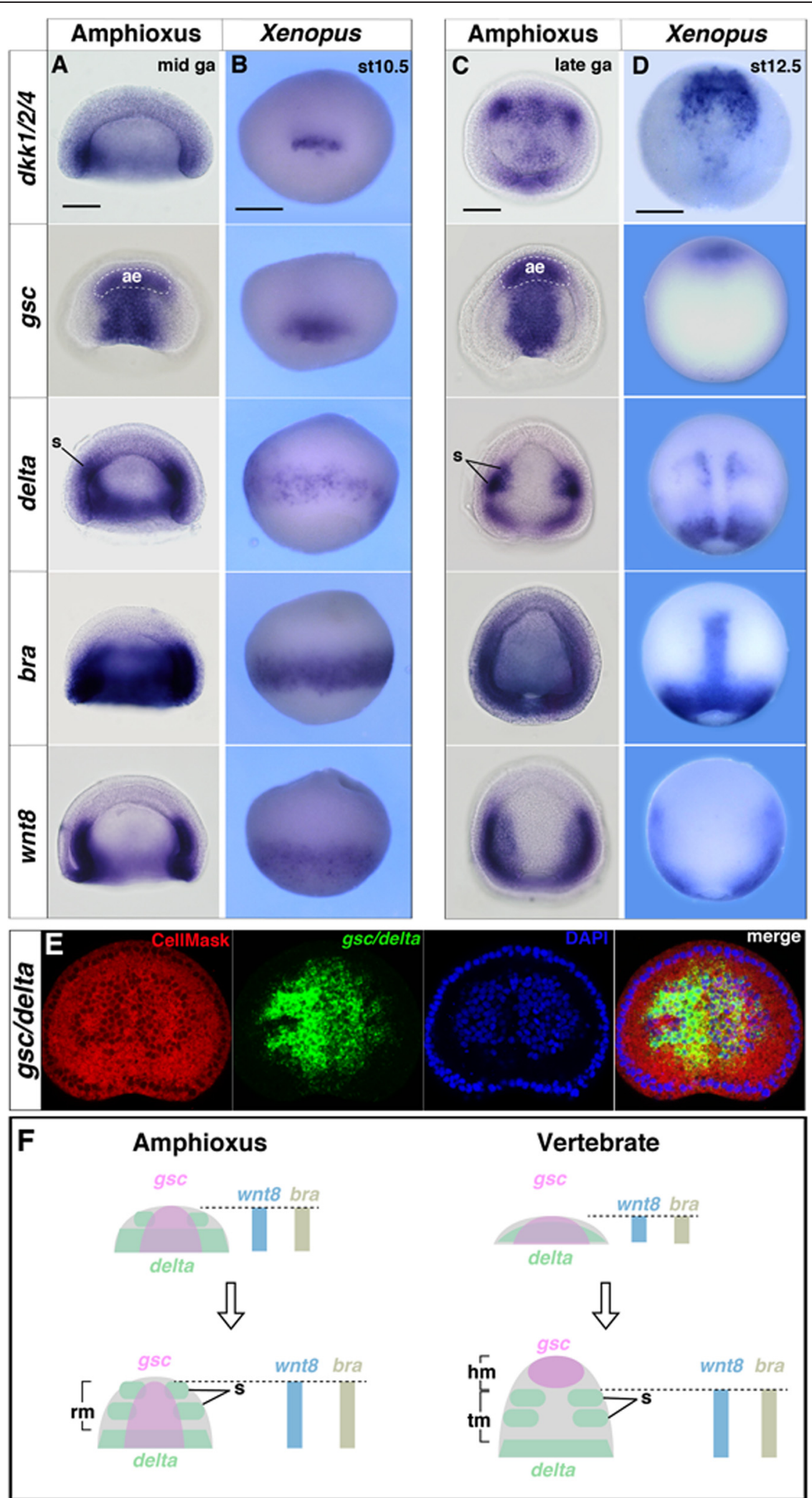

Fig. 2 (See legend on next page.) 
(See figure on previous page.)

Fig. 2 Comparison of regional gene expression patterns in the dorsal mesoderm of amphioxus and vertebrates. a-d In situ hybridization of (a) mid- and (c) late-gastrula amphioxus embryos and (b) stage 10.5 and (d) 12.5 Xenopus embryos for dkk1/2/4, gsc, delta, bra, and wnt8. Bfgsc expression was detected in the anterior endoderm that forms the foregut. ga gastrula, st stage, ae anterior endoderm, $s$ somite. e Fluorescence in situ hybridization of late gastrula amphioxus embryos stained with gsc/delta (Green). CellMask stained plasma membranes (red) and DAPI stained nuclei (blue). $\mathbf{f}$ Summary of dorsal mesoderm gene expression in amphioxus and vertebrates. Arrows indicate shifts of developmental stages from early- to late-gastrula stage. $\mathrm{rm}$ rostral mesoderm, $\mathrm{hm}$ head mesoderm, tm trunk mesoderm. Scale bars, $50 \mu \mathrm{m}$ in amphioxus, $500 \mu \mathrm{m}$ in Xenopus

Overlapping head and trunk marker gene expression was also detected based on the results of the dorsal marginal zone assay, suggesting that the effect of Xldd1 injection was not attributable to differences in the amount of yolk, but more likely to the loss of mesodermal cell movement (Additional file 1: Figure S4I-L).

These results are consistent with morphological changes observed at the tail bud stage in Xldd1-injected Xenopus embryos assimilated to an amphioxus-like condition. Specifically, the anterior-most somite, normally appearing just posterior to the anterior end of the notochord, was extended anteriorly into the prechordal domain (Fig. 3k-p). In these embryos, the somite marker $m y o D$ was expressed normally, similar to $m r f 1$ in amphioxus embryos (Fig. 3q-s). Interestingly, ectopic expression of $t b x 1$, a head mesoderm marker in vertebrates [10], was also detected in the Xldd1-injected Xenopus somites, similar to amphioxus $t b \times 1 / 10$, a somite marker in amphioxus [45], expression (Fig. 3t-w and Additional file 1: Figure S4G and H). These results suggest that the vertebrate-specific mesodermal involution during gastrulation is likely responsible for the A/P distinction between head and trunk mesoderm, which does not occur in amphioxus.

\section{A/P patterning in the dorsal mesoderm by different Wnt/ $\beta$-catenin-signalling input is a vertebrate novelty}

Previous functional studies in vertebrates have shown that the dorsal mesoderm is regionalized by a $\mathrm{Wnt} / \mathrm{\beta}$-catenin-signalling gradient along the $\mathrm{A} / \mathrm{P}$ axis during early embryogenesis [46]. The failure of $\mathrm{A} / \mathrm{P}$ segregation of mesodermal regional gene expression in Xldd1 mRNAinjected embryos suggests that $\mathrm{Wnt} / \beta$-catenin-signalling pathway control of these downstream genes is compromised in this context. We examined the nuclear localization of $\beta$-catenin, a downstream factor in the $\mathrm{Wnt} / \beta$-catenin signalling pathway, in Xldd1 mRNAinjected embryos. In the control embryos, nuclear localization of $\beta$-catenin was observed in the posterior dorsal mesodermal cells, but not in the anterior region (Fig. 4a-c). In Xldd1 mRNA-injected embryos, however, $\beta$-catenin localized to the nucleus in some cells, but there was no clear $\mathrm{A} / \mathrm{P}$ difference in the degree of nuclear localization (Fig. $4 \mathrm{~d}-\mathrm{f}$ ). The lack of obvious A/P difference in $\beta$-catenin nuclear localization was also observed in the amphioxus dorsal mesoderm (Fig. 4g-l).
Consistent with this result, a previous functional study showed that Wnt/ $\beta$-catenin signalling had no role in the $\mathrm{A} / \mathrm{P}$ patterning of the dorsal mesoderm during the gastrula stages [23]. These findings suggest that anterior low and posterior high $\mathrm{Wnt} / \beta$-catenin-signalling input is important in the segregation of regional marker genes of the dorsal mesoderm along the $\mathrm{A} / \mathrm{P}$ axis that evolved in vertebrates.

\section{Evolution of head mesoderm in vertebrates}

In this study, we propose that the vertebrate dorsal mesoderm evolved as an entirely novel pattern associated with a new mechanism of mesoderm specification. As was first described by Ernst Haeckel [47], amphioxus gastrulation involves a simple invagination similar to that in cnidarians, with little change in the spatial relationships between the ectoderm and mesoendoderm during development. Unlike in vertebrates, the dorsal mesoderm in amphioxus co-expresses both vertebrate head and trunk mesoderm marker genes (Fig. 5). Thus, the amphioxus dorsal mesoderm remains unspecified along the $\mathrm{A} / \mathrm{P}$ axis due to the absence of a vertebratespecific developmental program (Fig. 5). Vertebrate mesoderm, however, is uniquely polarized along the A/P axis into the head and trunk mesoderm based on mesodermal patterning mediated by vertebrate-specific cell movement. By the end of gastrulation, the head and trunk mesodermal identities are specified by anteroposteriorly dislocated expression of regional marker genes. This patterning mechanism also controls $\mathrm{A} / \mathrm{P}$ patterning of the overlying neuroectoderm. In vertebrates, the $\mathrm{A} / \mathrm{P}$ regional identity of the CNS is organized by vertical signals from the underlying dorsal mesoderm containing $\mathrm{A} / \mathrm{P}$ pattern information [48]. In amphioxus, based on the topography of regional markers, the CNS is patterned into domains largely comparable to the fore-/mid-/hindbrain and the spinal cord in vertebrate embryos [5]. However, three major signalling centres (anterior neural ridge, zona limitans intrathalamica, and midbrain-hindbrain boundary) are absent in the amphioxus CNS [5, 49]. Given that our current study indicated that the $\mathrm{A} / \mathrm{P}$ regional identity of the vertebrate dorsal mesoderm is fundamentally different from that of amphioxus (Fig. 5a), the three major signalling centres in the neuroectoderm of vertebrate embryos may have evolved through a reorganization of the dorsal mesoderm in an amphioxus-like chordate ancestor. 


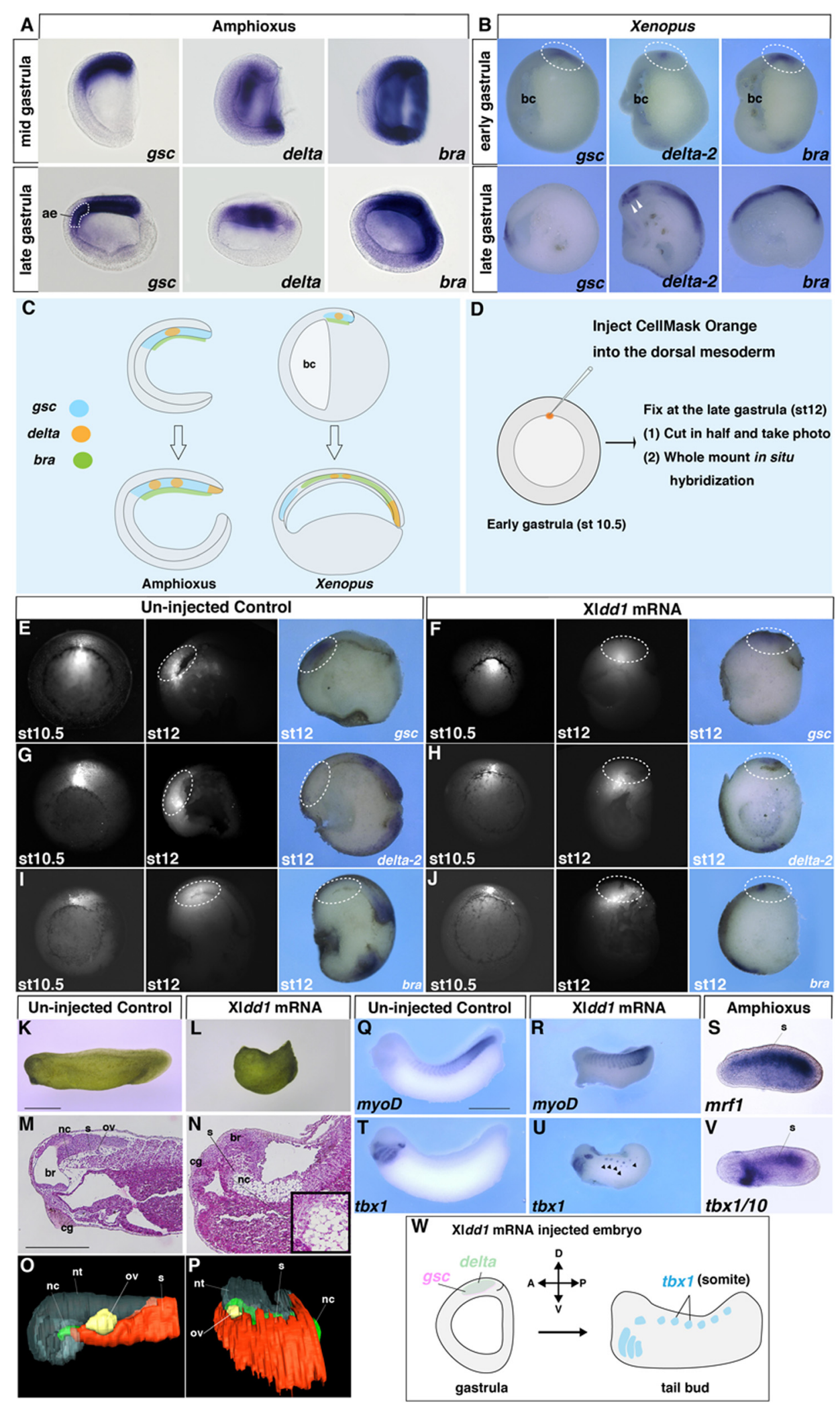

Fig. 3 (See legend on next page.) 


\begin{abstract}
(See figure on previous page.)
Fig. 3 Inhibition of mesodermal involution in vertebrates recapitulates amphioxus A/P patterning. In situ hybridization of gsc, delta and bra in amphioxus (a) and Xenopus (b) embryos. The arrowheads for delta-2 in the late gastrula Xenopus embryo indicate expression in somites. ae anterior endoderm, bc blastocoel. c Schematic diagram of relative size increase of the dorsal mesoderm during gastrulation in amphioxus and vertebrates. $\mathbf{d}$ Overview of the labelling study of the dorsal mesoderm in Xenopus. CellMask Orange (fluorescent dye) was injected into the dorsal mesoderm at stage (st) 10.5 and the embryos were cultured until stage 12. Fixed embryos were cut in half and then subjected to in situ hybridization. e, g, i At stage 12 in the control, labelled cells migrated anteriorly to form the head mesoderm $(n=21,100 \%)$ and expressed gsc $(n=2,100 \%)$ but not delta-2 ( $n=2$, $100 \%)$ or bra $(n=3,100 \%) . \mathbf{f}, \mathbf{h}, \mathbf{j}$ At stage 12 in the embryos that had been injected with $400 \mathrm{pg} / \mathrm{cell}$ XIdd $1 \mathrm{mRNA}$ at the four-cell blastomere stage, the labelled cells stayed near the blastopore region $(n=40,100 \%)$, and gsc $(n=2,100 \%)$, delta-2 $(n=6,100 \%)$, and bra $(n=3,100 \%)$ expression overlapped along the A/P axis. st stage. The dotted circle indicates the cells labelled by CellMask Orange. External morphology $(\mathbf{k}, \mathbf{I})$, histological sections $(\mathbf{m}, \mathbf{n})$ and 3-D reconstruction $(\mathbf{o}, \mathbf{p})$ of control $(n=22,100 \%$; left $)$ and XIdd 1 mRNA-injected embryos $(n=35$, $94 \%$; right). $n c$ notochord, $s$ somite, br brain, cg cement gland, ov otic vesicle, $n t$ neural tube. Expression of myoD (muscle differentiation marker) (q; $n=15,100 \%, \mathbf{r} ; n=14,100 \%)$ and tbx $1(\mathbf{t} ; n=12,100 \%, \mathbf{u} ; n=20,90 \%)$ in control and XIdd1 mRNA-injected Xenopus embryos, respectively. $\mathbf{s}$ mrfl and (v) tbx1/10 (pharynx and somite marker) expression in amphioxus embryos. w Schematic diagram of amphioxus-like developmental patterns in XIdd1 mRNA-injected Xenopus embryos. Scale bars, 1 mm (k, q) and $500 \mu m(\mathbf{m})$
\end{abstract}

Evolutionary reorganization of the entire dorsal mesoderm as described above would imply that individual amphioxus somites are not homologous to any specific region of the vertebrate head mesoderm. Our scenario instead favours the novel nature of the vertebrate mesoderm generated by modification in mesodermal patterning dynamics. From the perspective of vertebrate mesodermal specification, the segmented mesoderm in amphioxus appears as an intermediate between the head mesoderm and trunk somites, and vertebrate somites do not represent primitive traits, but rather derived traits established by removal of head mesoderm-like properties from ancestral somites. This scenario of vertebrate head evolution correlates with the observation that peripheral nerves in amphioxus possess traits of both cranial and spinal nerves [50, 51]. The mesodermal developmental pattern is shared among chordates only in the early gastrulae, in which the mesoderm is not yet anteroposteriorly polarized, possibly representing a plesiomorphic state (Fig. 5). The A/P patterning of mesodermal identities through the different $\mathrm{Wnt} / \beta$-catenin-signalling input takes place only in vertebrates later in the developmental process; along with unique changes in cell movement, this can be considered a synapomorphic developmental trait for this animal subphylum. As proposed by Haeckel, this indicates that the vertebrate body plan is established by recapitulating an amphioxus-like ancestral pattern (plesiomorphy) during the early-gastrula

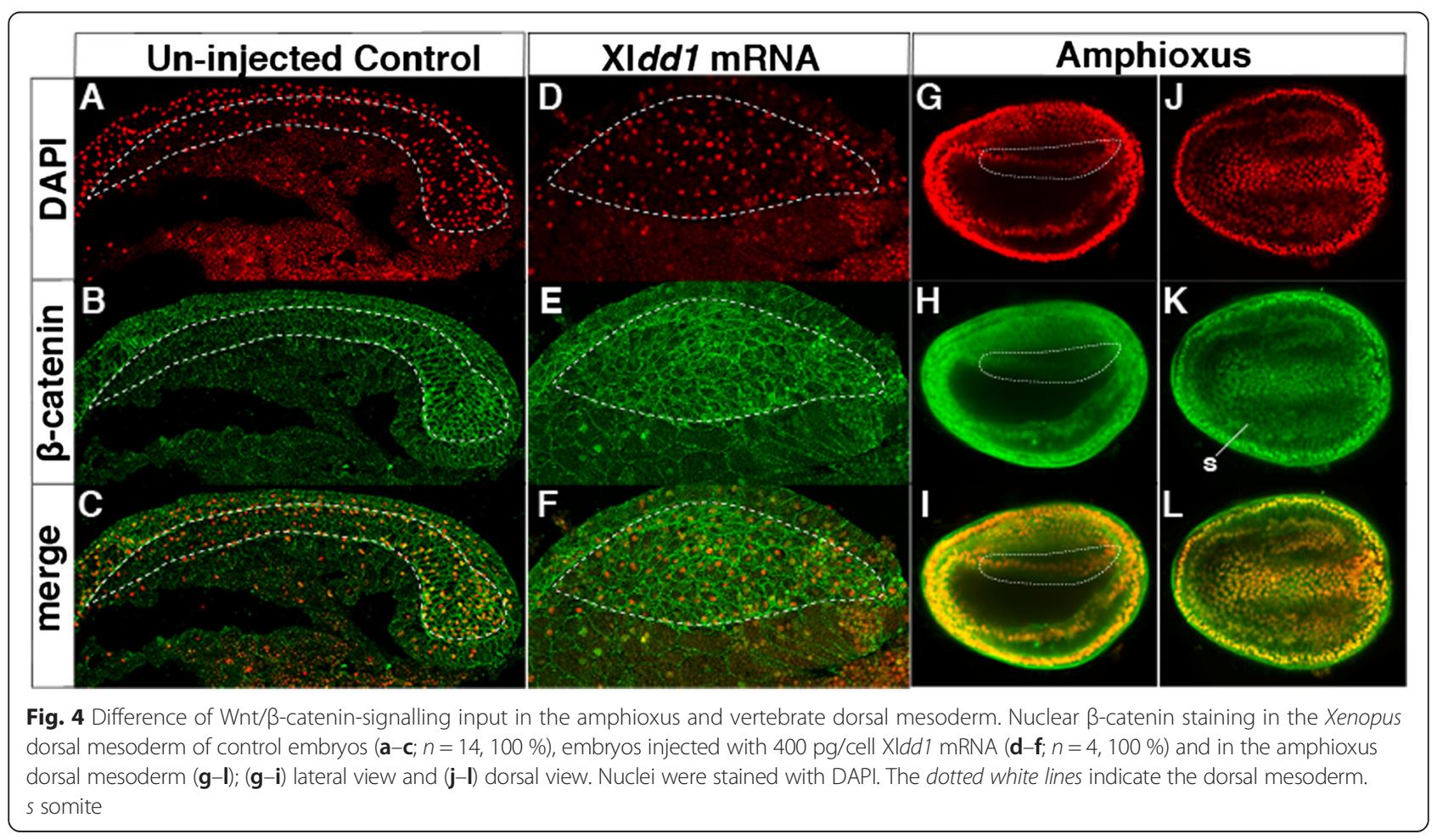




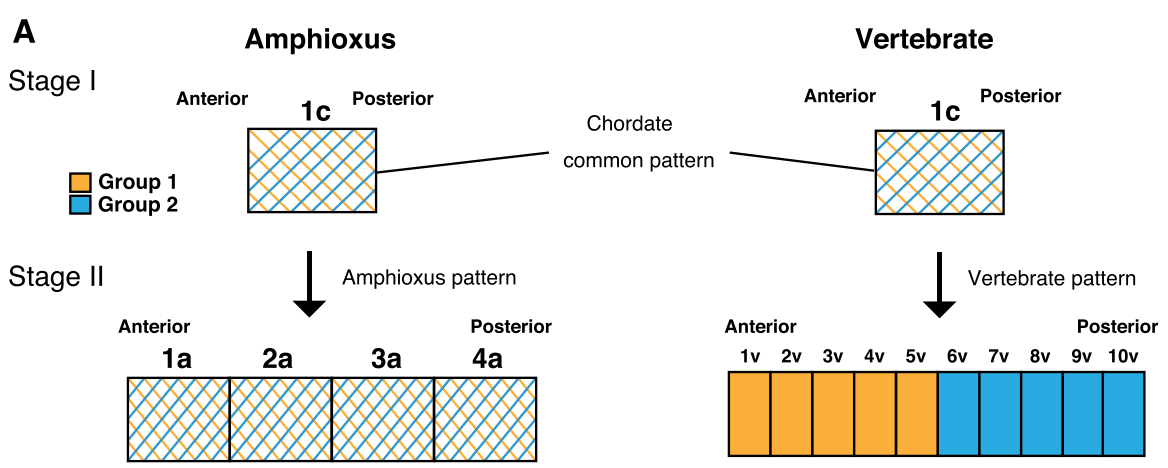

B

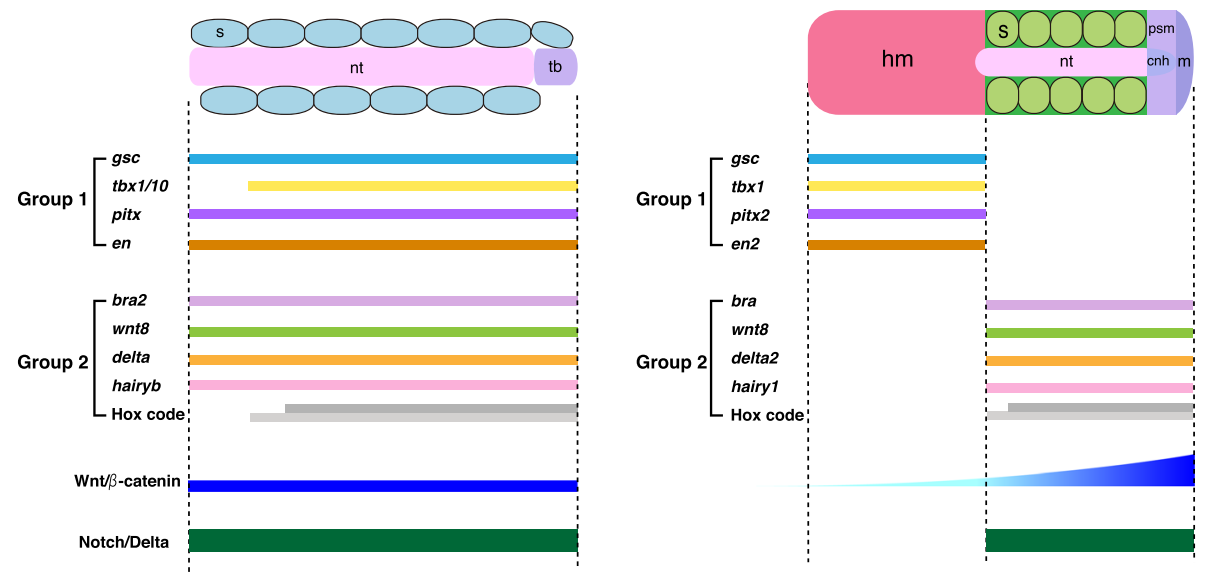

Fig. 5 Vertebrate head mesoderm evolved through polarization of ancestral mesodermal patterning. a In amphioxus and vertebrates, the dorsal mesoderm has two groups of genes that encode positional value (Group 1, orange; Group 2, light blue). At stage I (early-to mid-gastrula stages), group 1 and group 2 are co-expressed both in amphioxus and vertebrate dorsal mesoderm. At stage II (late-gastrula stage), group 1 genes shift anteriorly and group 2 genes shift posteriorly in vertebrates, whereas the amphioxus mesoderm retains the overlapped condition. Numbers indicate positional value of the dorsal mesoderm along the A/P axis. c chordate, $a$ amphioxus, $v$ vertebrates. $\mathbf{b}$ The dorsal mesoderm of vertebrates evolved by the reorganization of mesodermal genes under the control of Wnt/ $\beta$-catenin-signalling input. Notch/Delta signalling regulates somitogenesis both in amphioxus and vertebrates. s somite, $n t$ notochord, tb tail bud, psm presomitic mesoderm, cnh chordoneural hinge, $\mathrm{m}$ mesenchyme, $\mathrm{hm}$ head mesoderm. hairy genes are key components that regulate somite formation during development [16]. pitx2 and en 2 are expressed in head mesoderm of vertebrate embryos [10]

stage and engaging a novel pattern (synapomorphy) during the later stages. The comparison of mesodermal gene expression of vertebrates, amphioxus, and hemichordates (as an out-group taxon) suggests that the amphioxus mesoderm has an intermediate nature, possibly representing a plesiomorphic state for deuterostomes (http:// www.ibiology.org/ibioseminars/evolution-ecology/marc-wkirschner-part-3.html). This characterization of the evolutionary sequence of developmental dynamics in chordates also provides insight into the potential origins of mesoderm and mesodermal segments in bilaterians.

\section{Possible mechanism of mesodermal involution unique to vertebrates}

In this study, we showed that inhibition of mesodermal involution in vertebrate embryos recapitulated amphioxus development (Fig. 3). The lack of mesodermal involution and likely convergent extension in amphioxus gastrulation indicates that the developmental program for mesodermal involution in vertebrates is absent in amphioxus. Disruption of cadherin-mediated cell-cell adhesion is essential during mesodermal involution and convergent extension in vertebrates, and fibronectin leucine-rich-repeat transmembrane 3 (Flrt3) and a small GTPase (Rnd1) control C-cadherin degradation $[26,34,52,53]$. A BLAST search revealed a homologue of rnd1 in amphioxus, but not of flrt3 (Additional file 1: Figure S5A and B). In amphioxus, rnd1 expression was observed around the blastopore (Additional file 1: Figure S6A-H). However, overexpression of Bfrnd1 mRNA could not rescue the loss of endogenous rnd 1 in Xenopus (Additional file 1: Figure S6I-M). These findings suggest that involvement of the cadherin degradation system in mesodermal involution as well 
as convergence and extension may have emerged in the vertebrate lineage.

\section{Conclusions}

Our findings indicate that the $\mathrm{A} / \mathrm{P}$ patterning of the vertebrate dorsal mesoderm evolved from an amphioxuslike ancestral mesoderm through $\mathrm{A} / \mathrm{P}$ polarization of mesodermal specification to divide into the unsegmented head mesoderm anteriorly and the segmented trunk somites posteriorly. Vertebrate head mesoderm is thus an evolutionary novelty.

\section{Additional file}

Additional file 1: Figure S1. Dorsal mesoderm formation in chordates. Figure S2. Phylogenetic trees of dorsal mesoderm genes. Figure S3.

Dorsal mesodermal gene expression in lamprey (L. japonicum) and shark (S. torazame) embryos. Figure S4. Suppression of the Wnt/PCP-signaling pathway in Xenopus embryos. Figure S5. Flrt3 evolved in the vertebrate lineage. Figure S6. Flrt3-Rnd1 system is essential for mesoderm formation in vertebrates. Table S1. Summary of expression pattern of genes in Figure S2. (DOCX $8727 \mathrm{~kb})$

\section{Competing interests}

The authors declare they have no competing interests.

\section{Authors' contributions}

TO and SK wrote the paper. TO and TH performed the experiments. TO, TA and $\mathrm{HI}$ designed the experiments. All authors read and approved the final manuscript.

\section{Acknowledgements}

We thank Linda Holland and Nick Holland of the University of California, San Diego, CA, USA for help with the collection of amphioxus and for discussion. We also thank Yasuhisa Henmi of Kumamoto University, Japan and Kinya Yasui of Hiroshima University, Japan for helping with amphioxus collection and Shigehiro Kuraku of RIKEN, Japan for preparing the phylogenetic trees. This research was supported by a KAKENHI Grant-in-Aid for Young Scientists (B) from the Japan Society for the Promotion of Science (grant number 24770222)

\section{Author details}

${ }^{1}$ Kuratani Evolutionary Morphology Laboratory, RIKEN Center for Developmental Biology, 2-2-3 Minatojima-minamimachi, Chuo-ku, Kobe 650-0047, Japan. ²Pattern Formation Group, Graduate School of Frontier Biosciences, Osaka University, 1-3 Yamadaoka, Suita, Osaka 565-0871, Japan. ${ }^{3}$ Laboratory for Axial Pattern Dynamics, RIKEN Center for Developmental Biology, 2-2-3 Minatojima-minamimachi, Chuo-ku, Kobe 650-0047, Japan.

Received: 9 June 2015 Accepted: 22 September 2015

Published online: 09 November 2015

\section{References}

1. Gans C, Northcutt RG. Neural crest and the origin of vertebrates: a new head. Science. 1983;220:268-74.

2. Onai T, Irie N, Kuratani S. The evolutionary origin of the vertebrate body plan: the problem of head segmentation. Annu Rev Genom Hum Genet. 2014;15:443-59.

3. Romer AS. The vertebrate as a dual animal-somatic and visceral. Evol Biol. 1972:6:121-56.

4. Holland LZ. Chordate roots of the vertebrate nervous system: expanding the molecular toolkit. Nat Rev Neurosci. 2009;10(10):736-46.

5. Holland LZ, Carvalho JE, Escriva H, Laudet V, Schubert M, Shimeld SM, et al. Evolution of bilaterian central nervous systems: a single origin? EvoDevo. 2013;4(1):27
6. Wicht H, Lacalli TC. The nervous system of amphioxus: structure, development, and evolutionary significance. Can J Zool. 2005;83:122-50.

7. Holland LZ, Onai T. Early development of cephalochordate (amphioxus). WIREs Dev Biol. 2011;1:167-83.

8. Holland LZ, Holland ND, Gilland E. Amphioxus and the evolution of head segmentation. Integr Comp Biol. 2008;48(5):630-46.

9. Holland LZ, Kene M, Williams NA, Holland ND. Sequence and embryonic expression of the amphioxus engrailed gene (AmphiEn): the metameric pattern of transcription resembles that of its segment-polarity homolog in Drosophila. Development. 1997;124(9):1723-32.

10. Adachi N, Takechi M, Hirai T, Kuratani S. Development of the head and trunk mesoderm in the dogfish, Scyliorhinus torazame: II. Comparison of gene expression between the head mesoderm and somites with reference to the origin of the vertebrate head. Evol Dev. 2012;14(3):257-76.

11. Kazanskaya O, Glinka A, Niehrs C. The role of Xenopus dickkopf1 in prechordal plate specification and neural patterning. Development. 2000;127(22):4981-92.

12. Niehrs C. Regionally specific induction by the Spemann-Mangold organizer. Nat Rev Genet. 2004;5(6):425-34

13. Cho KW, Blumberg B, Steinbeisser H, De Robertis EM. Molecular nature of Spemann's organizer: the role of the Xenopus homeobox gene goosecoid. Cell. 1991;67(6):1111-20.

14. Smith JC, Price BM, Green JB, Weigel D, Herrmann BG. Expression of a Xenopus homolog of Brachyury (T) is an immediate-early response to mesoderm induction. Cell. 1991;67(1):79-87.

15. Peres JN, McNulty CL, Durston AJ. Interaction between X-Delta-2 and Hox genes regulates segmentation and patterning of the anteroposterior axis. Mech Develop. 2006;123(4):321-33.

16. Pourquie O. Vertebrate segmentation: from cyclic gene networks to scoliosis. Cell. 2011;145(5):650-63.

17. Martin BL, Kimelman D. Regulation of canonical Wnt signaling by Brachyury is essential for posterior mesoderm formation. Dev Cell. 2008;15(1):121-33.

18. Morley RH, Lachani K, Keefe D, Gilchrist MJ, Flicek P, Smith JC, et al. A gene regulatory network directed by zebrafish No tail accounts for its roles in mesoderm formation. Proc Natl Acad Sci U S A. 2009;106(10):3829-34.

19. Aulehla A, Wiegraebe W, Baubet V, Wahl MB, Deng C, Taketo M, et al. A beta-catenin gradient links the clock and wavefront systems in mouse embryo segmentation. Nature Cell Biol. 2008;10(2):186-93.

20. Holland PW, Koschorz B, Holland LZ, Herrmann BG. Conservation of Brachyury (T) genes in amphioxus and vertebrates: developmental and evolutionary implications. Development. 1995;121(12):4283-91.

21. Yu JK, Satou Y, Holland ND, Shin IT, Kohara Y, Satoh N, et al. Axial patterning in cephalochordates and the evolution of the organizer. Nature. 2007:445(7128):613-7.

22. Rasmussen SL, Holland LZ, Schubert M, Beaster-Jones L, Holland ND. Amphioxus AmphiDelta: evolution of Delta protein structure, segmentation, and neurogenesis. Genesis. 2007;45(3):113-22.

23. Onai T, Takai A, Setiamarga DH, Holland LZ. Essential role of Dkk3 for head formation by inhibiting Wnt/beta-catenin and Nodal/Ng1 signaling pathways in the basal chordate amphioxus. Evol Dev. 2012;14(4):338-50.

24. Zhang S, Holland ND, Holland LZ. Topographic changes in nascent and early mesoderm in amphioxus embryos studied by Dil labeling and by in situ hybridization for a Brachyury gene. Dev Genes Evol. 1997;206:532-5.

25. Damas H. Recherches sur le dévellopement de Lampetra fluviatilis L. Contribution à l'étude de la Céphalogenèse des vertébrés. Archives de Biologie, Liége et Paris. 1944;55:1-248.

26. Hammerschmidt M, Wedlich D. Regulated adhesion as a driving force of gastrulation movements. Development. 2008;135(22):3625-41.

27. Winklbauer R, Damm EW. Internalizing the vegetal cell mass before and during amphibian gastrulation: vegetal rotation and related movements. WIREs Interdiscip Rev Dev Biol. 2012;1:301-6.

28. Holland LZ, Onai T. Analyses of gene function in amphioxus embryos by microinjection of mRNAs and morpholino oligonucleotides. Methods Mol Biol. 2011;770:423-38.

29. Takio Y, Kuraku S, Murakami Y, Pasqualetti M, Rijli FM, Narita Y, et al. Hox gene expression patterns in Lethenteron japonicum embryos-insights into the evolution of the vertebrate Hox code. Dev Biol. 2007;308(2):606-20.

30. Ballard WW, Mellinger J, Lechenaud $H$. A series of normal stages for development of Scyliorhinus canicula, the lesser spotted dogfish (Chondrichtyes: Scyliorhinidae). J Exp Zool. 1993;267:318-36. 
31. Onai T, Matsuo-Takasaki M, Inomata $H$, Aramaki T, Matsumura M, Yakura R, et al. XTsh3 is an essential enhancing factor of canonical Wnt signaling in Xenopus axial determination. EMBO J. 2007;26(9):2350-60.

32. Butler K, Zorn AM, Gurdon JB. Nonradioactive in situ hybridization to xenopus tissue sections. Methods. 2001;23(4):303-12.

33. Nieuwkoop PD, Faber J. Normal table of Xenopus laevis. Amsterdam, Netherlands: North-Holland Publishing Co; 1956.

34. Ogata S, Morokuma J, Hayata T, Kolle G, Niehrs C, Ueno N, et al. TGF-beta signaling-mediated morphogenesis: modulation of cell adhesion via cadherin endocytosis. Genes Dev. 2007;21(14):1817-31.

35. Lu TM, Luo YJ, Yu JK. BMP and Delta/Notch signaling control the development of amphioxus epidermal sensory neurons: insights into the evolution of the peripheral sensory system. Development. 2012;139(11):2020-30.

36. Tamura K, Peterson D, Peterson N, Stecher G, Nei M, Kumar S. MEGA5 molecular evolutionary genetics analysis using maximum likelihood, evolutionary distance, and maximum parsimony methods. Mol Biol Evol. 2011;28(10):2731-9.

37. Keller R. Mechanisms of elongation in embryogenesis. Development. 2006;133(12):2291-302.

38. Shi J, Keller R. Gastulation in Xenopus laevis: involution-a current view Semin Cell Dev Biol. 1994:5:85-90.

39. Keller R, Davidson L, Edlund A, Elul T, Ezin M, Shook D, et al. Mechanisms of convergence and extension by cell intercalation. Philos Trans R Soc Lond B Biol Sci. 2000;355(1399):897-922.

40. Habas R, Kato Y, He X. Wnt/Frizzled activation of Rho regulates vertebrate gastrulation and requires a novel Formin homology protein Daam1. Cell. 2001;107(7):843-54.

41. Solnica-Krezel L, Sepich DS. Gastrulation: making and shaping germ layers. Annu Rev Cell Dev Biol. 2012;28:687-717.

42. Sokol SY. Analysis of Dishevelled signalling pathways during Xenopus development. Curr Biol. 1996;6(11):1456-67.

43. Tanegashima K, Zhao H, Dawid IB. WGEF activates Rho in the Wnt-PCP pathway and controls convergent extension in Xenopus gastrulation. EMBO J. 2008:27(4):606-17.

44. Dzamba BJ, Jakab KR, Marsden M, Schwartz MA, DeSimone DW. Cadherin adhesion, tissue tension, and noncanonical Wnt signaling regulate fibronectin matrix organization. Dev Cell. 2009;16(3):421-32.

45. Mahadevan NR, Horton AC, Gibson-Brown JJ. Developmental expression of the amphioxus $\mathrm{Tbx} 1 / 10$ gene illuminates the evolution of vertebrate branchial arches and sclerotome. Dev Genes Evol. 2004;214(11):559-66.

46. De Robertis EM. Evo-devo: variations on ancestral themes. Cell. 2008;132(2):185-95.

47. Haeckel $E$. The evolution of man [English translation of third edition of Anthropogenie]. 3rd ed. New York: Fowle; 1876

48. Sharpe CR, Gurdon JB. The induction of anterior and posterior neural genes in Xenopus laevis. Development. 1990;109(4):765-74.

49. Pani AM, Mullarkey EE, Aronowicz J, Assimacopoulos S, Grove EA, Lowe CJ. Ancient deuterostome origins of vertebrate brain signalling centres. Nature. 2012:483(7389):289-94.

50. Fritzsch B, Northcutt RG. Cranial and spinal nerve organization in amphioxus and lampreys: evidence for an ancestral craniate pattern. Acta Anat (Basel). 1993;148(2-3):96-109.

51. Kuratani S. Spatial distribution of postotic crest cells defines the head/trunk interface of the vertebrate body: embryological interpretation of peripheral nerve morphology and evolution of the vertebrate head. Anat Embryol (Berl). 1997:195(1):1-13.

52. Egea J, Erlacher C, Montanez E, Burtscher I, Yamagishi S, Hess M, et al. Genetic ablation of FLRT3 reveals a novel morphogenetic function for the anterior visceral endoderm in suppressing mesoderm differentiation. Genes Dev. 2008;22(23):3349-62

53. Fodor E, Zsigmond A, Horvath B, Molnar J, Nagy I, Toth G, et al. Full transcriptome analysis of early dorsoventral patterning in zebrafish. PLoS One. 2013:8(7):e70053

\section{Submit your next manuscript to BioMed Central and take full advantage of:}

- Convenient online submission

- Thorough peer review

- No space constraints or color figure charges

- Immediate publication on acceptance

- Inclusion in PubMed, CAS, Scopus and Google Scholar

- Research which is freely available for redistribution

Submit your manuscript at www.biomedcentral.com/submit 